\title{
How to Correct a Task Error: Task-Switch Effects Following Different Types of Error Correction
}

\author{
Marco Steinhauser \\ University of Konstanz
}

\begin{abstract}
It has been proposed that switch costs in task switching reflect the strengthening of task-related associations and that strengthening is triggered by response execution. The present study tested the hypothesis that only task-related responses are able to trigger strengthening. Effects of task strengthening caused by error corrections were considered. Recent findings suggest that errors lead to erroneous task strengthening, which, however, can be reversed by immediate error correction (Steinhauser \& Hübner, 2006). In three experiments, the present study examined whether this effect is also obtained when task responses and correction responses share the same response categories but are assigned to different hands or different response modalities (manual vs. vocal). Results indicated that only corrections with the same hand but not corrections with the alternative hand or a different response modality can reverse erroneous task strengthening. These results suggest that only the execution of task-related responses triggers task strengthening, whereas the activation of task-related response categories is not sufficient.
\end{abstract}

Keywords: executive control, task switching, error processing, automatization

In recent years, the task-switching paradigm (Allport, Styles, \& Hsieh, 1994; Meiran, 1996; Rogers \& Monsell, 1995) has become a popular method for investigating control processes and task conflicts. The paradigm requires that two or more tasks be performed in random or prespecified order. When performance on task-switch trials is compared with that on task-repetition trials, then so-called switch costs are obtained. Whereas some accounts have assumed that the costs reflect control processes involved in switching the task set (Meiran, 1996; Rogers \& Monsell, 1995), others have suggested that at least a portion of the cost is due to memory processes such as priming or associative strengthening of tasks (e.g., Allport et al., 1994). One specific account assumed that task execution leads to strengthening of the current task but weakening of competing tasks (e.g., Meiran, 2000). Accordingly, switch costs emerge because repeating the previously strengthened task is beneficial, whereas switching toward the previously weakened task is costly. Recent studies have highlighted particularly the role of response execution for task strengthening (Philipp, Jolicoeur, Falkenstein, \& Koch, 2007; Schuch \& Koch, 2003; Steinhauser \& Hübner, 2006). For instance, Steinhauser and Hübner (2006) proposed a response-based strengthening mechanism, which implies that, whenever a response is executed that is part of a task's response set, then all active task rules are strengthened by means of Hebbian learning.

Marco Steinhauser, University of Konstanz, Konstanz, Germany.

This research was supported by a grant from the Deutsche Forschungsgemeinschaft (DFG: Ste 1708/1-1). I am grateful to Marleen Vogt, Tanja Beyer, and Roman Fröde for assistance in conducting the experiments, and to Nachshon Meiran for comments on an earlier version of this article.

Correspondence concerning this article should be addressed to Marco Steinhauser, University of Konstanz, Department of Psychology, Fach D29, D-78457 Konstanz, Germany. E-mail: Marco.Steinhauser@ uni-konstanz.de
The present study investigated the role of response execution in more detail. I report on three experiments that show that it is, indeed, the execution of a task-relevant response and not only the selection of an abstract response category that triggers strengthening. As discussed later in this article, this finding suggests that the mechanism underlying the switch costs plays a role in the automatization of effector-specific cognitive skills.

Initial evidence for a role of the response for the occurrence of switch costs has been provided by studies using a no-go procedure. Schuch and Koch (2003) instructed participants to withhold the response whenever a no-go signal appeared. It turned out that the switch costs were eliminated after these no-go trials. Initially, Schuch and Koch took this as evidence that response selection is necessary for subsequent switch costs to occur. However, a follow-up study by Philipp et al. (2007) improved the no-go method by introducing trials with delayed go/no-go signals, which allowed response selection but not response execution to occur. The fact that switch costs were absent even following these trials led to the conclusion that it is the execution of an overt response that is necessary to produce switch effects.

Further evidence came from studies showing that switch costs turned into switch benefits after trials on which an error had occurred (Steinhauser \& Hübner, 2006, 2008). This finding can be explained by assuming that a portion of errors resulted from task confusions (see also Meiran \& Daichman, 2005) and that the accidental execution of the wrong task implied that the wrong task was strengthened. As a consequence, a subsequent switch to the erroneously applied task became beneficial, whereas a repetition of the previously intended task became costly. However, this result alone does not imply that strengthening is triggered by response execution.

Rather, this conclusion can be derived from examining the effects of different types of error-detection responses on the switch costs. When participants were required to signal their errors im- 
mediately by pressing a neutral key (Steinhauser \& Hübner, 2006 , Experiment 2), then even these signaled errors were followed by switch benefits. This outcome indicates that even when an error is consciously detected, the wrong task is strengthened. However, when participants were required to correct their errors immediately by pressing the correct response (Steinhauser \& Hübner, 2006, Experiment 3), then these corrected errors were followed by switch costs. Obviously, error correction (but not error signaling) triggered strengthening of the correct task, which turned the switch benefits back into switch costs. From these results, it was concluded that only the execution of a task-relevant response triggers task strengthening. Because both error signaling and error correction should require that the correct response as well as the correct task becomes activated after the error (Steinhauser, Maier, \& Hübner, 2008), it was further concluded that merely activating a task does not imply that this task is strengthened.

From this reasoning, one could infer that only responses from the task's response set can act as a trigger. However, the question emerges as to what exactly defines the response set. On the one hand, it could be defined with respect to the actual responses. For instance, when a task requires that a left and right button is pressed with two fingers of the right hand, then only the execution of these two responses would trigger task strengthening. On the other hand, the response set could be defined with respect to abstract response categories. In this example, task strengthening could be triggered when a motor plan is executed that involves a "left response."

Indeed, there is evidence that abstract response categories play a role under task-switching conditions. For instance, response repetition effects are obtained when the response is repeated as well as when the response category is repeated (Hübner \& Druey, 2006; Schuch \& Koch, 2004). Moreover, Hübner and Druey (2006) showed that it is sufficient that a response category is activated for these repetition effects to occur, whereas the actual execution is not necessary. Indeed, mere activation of abstract response categories could also account for the results found by Steinhauser and Hübner (2006): Error signaling did not involve only a different response as the main task, it also did not involve the same response categories as the main task.

In the present study I tested whether it is only the execution of a task-relevant response that triggers task strengthening. Three task-switching experiments were conducted in which participants randomly alternated between two simple numerical judgments (odd/even; less than/greater than 5). A deadline procedure was applied to induce errors, and participants were required to correct these errors immediately. In Experiments 1 and 2, two types of error correction were compared. Whereas task responses were always given with a left and right finger of one hand, error corrections were given either with the left and right finger of the same hand (same-hand corrections) or with those of the other hand (hand-switch corrections). Accordingly, hand-switch corrections involved the same response categories (left, right) as the task responses but different effectors than the task responses. If activating task-relevant response categories is sufficient to trigger task strengthening, then switch costs should be obtained following corrected errors even when error corrections require an effector switch. In contrast, if only the execution of an actual task response triggers strengthening, then switch benefits should be obtained following hand-switch corrections but switch costs following same-hand corrections. Whereas the first experiment directly com- pared the effects of the two correction types, the second experiment compared hand-switch corrections with uncorrected errors. Experiment 3 examined whether task strengthening is triggered by error corrections involving a modality switch. Whereas the main task required the same manual responses as those in the previous experiments, errors had to be corrected by vocalizing the correct response category ("left" or "right"). If only task-relevant responses can trigger strengthening, vocally corrected errors also should be followed by switch benefits.

\section{Experiment 1}

\section{Method}

Participants. Twenty-four participants (15 women, 9 men) between 18 and 28 years of age (mean age 23.2 years) with normal or corrected-to-normal vision participated in the study. Participants were recruited at the University of Konstanz and were paid $€ 5$ per hour.

Apparatus. The stimuli were presented on a 21 -inch color monitor. An IBM-compatible PC controlled stimulus presentation and response registration. Responses were given using a German standard keyboard (for simplicity, the description refers to the American keyboard layout).

Stimuli. Stimuli were the digits $1,3,6$, and 8 with a width of $1.24^{\circ}$ visual angle and a height of $1.77^{\circ}$ visual angle at a viewing distance of $127 \mathrm{~cm}$. Only those stimuli were used that required different responses for the two tasks because only for these stimuli could a task confusion lead to an error (Steinhauser \& Hübner, 2006). A circle and a square of the same size were used as cues. Cues and stimuli were presented in white color on a black background.

Design and procedure. On each trial, participants had to apply one of two judgments to a digit presented on the screen. In the parity judgment, the digit had to be classified as odd or even. In the magnitude judgment, the digit had to be classified as less than five or greater than five. Half of the participants gave their task responses with the left hand, and the other half gave their task responses with the right hand. A left key ( $Z$ key with the middle finger of the left hand or 1 key on the number pad with the index finger of the right hand) had to be pressed for the categories even and less than five, and a right key ( $X$ with the index finger of the left hand or 2 key on the number pad with the middle finger of the right hand) had to be pressed for the categories odd and greater than five.

Participants were instructed to correct errors immediately, either by giving the correct response with the same hand as that used for the main tasks (same-hand correction condition) or by giving the correct response with the hand not used for the main tasks (handswitch correction condition). For instance, if the parity of the digit 3 was erroneously classified by pressing the left key with the right hand, then this error had to be corrected either by pressing the right key with the right hand (if same-hand corrections were required) or the right key with the left hand (if hand-switch corrections were required). In the same-hand correction condition, the hand not used for the main tasks was removed from the keys.

Each trial started with the presentation of the cue for $300 \mathrm{~ms}$ followed by a blank screen for $900 \mathrm{~ms}$. The stimulus was presented for $150 \mathrm{~ms}$ followed by a blank screen. After a specific interval, an 
acoustical deadline signal $(800 \mathrm{~Hz}, 150 \mathrm{~ms})$ was provided, and participants were instructed to respond before this signal appeared. A new trial started $1,000 \mathrm{~ms}$ after the response. When a correction response occurred during this interval, another interval of $1,000 \mathrm{~ms}$ Was started. No feedback on the accuracy of the response was provided.

Participants worked through 20 test blocks of 64 trials, resulting in a total of 1,280 trials. Within each block, the order of judgments was randomized. In half of the blocks, participants were instructed to correct their errors using same-hand corrections. In the other half, hand-switch corrections were required. Blocks with samehand corrections and hand-switch corrections alternated, and the condition of the first block was counterbalanced across participants. The blocks were distributed on two experimental sessions. These sessions were preceded by a practice session without error correction, in which the deadline was adjusted according to the method described in Steinhauser and Hübner (2006)

\section{Results and Discussion}

The data were analyzed in the same way as in Steinhauser and Hübner (2006). ' Trials were excluded in which more than one correction response with one hand was given or in which a response occurred during the cue-stimulus interval $(<1 \%)$. In addition, to compute response times only, I excluded trials with response times less than or greater than three standard deviations below or above the mean computed for each condition and each participant $(1.2 \%)$. For statistical analysis, error rates were transformed using an arcsine transformation (Winer, Brown, \& Michels, 1991).

Table 1 shows frequencies, response times, and correction latencies for different trial types. Remarkably, there was a considerable number of spontaneous same-hand corrections even when this was not instructed (about $30 \%$ of all errors in the hand-switch condition). This finding indicates that the rate of automatic corrections is especially high when an experiment contains conditions with instructed corrections (Fiehler, Ullsperger, \& Von Cramon, 2005; Steinhauser \& Huibner, 2006; Steinhauser et al., 2008). This outcome could reflect that instructed error correction in some blocks induces increased performance monitoring in all blocks, which leads to spontaneous and unintended corrections. Apart from that, participants reliably adhered to the different correction instructions. The majority of errors were correctly corrected in the same-hand correction condition $(88.0 \%)$ as well as in the handswitch correction condition (66.8\%, including trials with an additional spontaneous correction). Only a few errors were corrected by pressing the wrong key $(4.9 \%)$ or were not corrected at all $(16.4 \%)$.

To test my hypothesis, I included only certain trial types in the analyses. From the same-hand correction condition, I included only trials following uncorrected correct responses $(n-1$ correct) and trials following errors corrected by a single same-hand correction ( $n-1$ error). From the hand-switch correction condition, I included trials following uncorrected correct responses $(n-1$ correct) and trials following errors corrected by a single handswitch correction ( $n-1$ error). Response times of correct responses and error rates were entered into a three-way analysis of variance (ANOVA) with repeated measures on the variables correction condition (same-hand correction, hand-switch correction), task transition (repetition, switch), and $n-1$ accuracy ( $n-1$ correct, $n-1$ error). ${ }^{2}$ The results are shown in Figure 1. For the error rates, the three-way interaction between all three variables was significant, $F(1,23)=6.12, p<.05$. To reveal the source of this interaction, the contrast representing the interaction between the variables task transition and $n-1$ accuracy was computed separately for each correction condition. For the hand-switch correction condition, the interaction reached significance, $F(1,11)=$ $20.67, p<.001$. Whereas switch costs were obtained following correct responses (repetition: $14.2 \%$; switch: $21.3 \%$ ), switch benefits were obtained following errors with hand-switch corrections (repetition: $20.5 \%$; switch: $16.5 \%$ ). This contrast was not significant for the same-hand correction condition $(F<3.00)$. Similar switch costs were found following correct responses (repetition: 15.0\%; switch: $23.2 \%$ ) and following errors with same-hand corrections (repetition: 16.8\%; switch: $21.9 \%$ ). For the response times, only a marginally significant three-way interaction between correction condition, task transition, and $n-1$ accuracy was obtained, $F(1,23)=3.93, p<.10$. However, Figure 1 shows that the response times exhibit a trend toward a result similar to that in the error rates.

Taken together, these data revealed that only errors corrected by same-hand corrections led to switch costs on subsequent trials. In contrast, errors corrected by hand-switch corrections still showed switch benefits typically observed after uncorrected errors. This finding indicates that only same-hand corrections and not handswitch corrections are sufficient to trigger strengthening of the correct task. As a further test of this hypothesis, a second experiment was conducted in which the effects of hand-switch corrections were directly compared with the effects of uncorrected errors. If hand-switch corrections imply no strengthening at all, the effects of hand-switch corrections should be similar to those of uncorrected errors.

\section{Experiment 2}

\section{Method}

Twenty-two participants ( 18 women, 4 men) between 18 and 33 years of age (mean age 22.1 years) with normal or corrected-tonormal vision participated in the study. The design and procedure of the experiment were the same as in Experiment 1 with one exception: The same-hand correction condition was replaced with a no-correction condition. In this condition, participants were instructed not to correct their errors.

\footnotetext{
${ }^{1}$ In Steinhauser and Hübner (2006), stimulus repetitions were excluded to demonstrate that error strengthening occurred on the level of tasks and not on the level of stimulus-response associations. In the present study, 1 report only analyses including stimulus repetitions. However, the results of this and the following experiments remained the same when stimulus repetitions were excluded.

${ }^{2}$ In each ANOVA and for each contrast, the variable response transition (response repetition, response alternation) was included to guarantee that results were equally affected by both trial types. For brevity, effects involving this variable are not reported. However, there was no significant interaction between this variable and any of the effects of interest.
} 
Table 1

Relative Frequencies, Mean Response Times, and Correction Latencies of Trials With Different Correction Types for Errors and Correct First Responses Within the Same-Hand Condition and Hand-Switch Condition, Experiment 1

\begin{tabular}{|c|c|c|c|c|c|c|}
\hline \multirow[b]{2}{*}{ Correction } & \multicolumn{3}{|c|}{ Correct } & \multicolumn{3}{|c|}{ Error } \\
\hline & Frequency & RTI & RT2 & Frequency & RTI & $\mathrm{RT} 2$ \\
\hline \multicolumn{7}{|l|}{ Same hand } \\
\hline No correction & $98.9(0.1)$ & $379(16)$ & & $12.0(3.4)$ & $539(103)^{\mathrm{a}}$ & \\
\hline Instructed correction & $1.1(0.1)$ & $409(53)^{\mathrm{l}}$ & $647(110)^{\mathrm{b}}$ & $88.0(3.4)$ & $365(21)$ & $442(27)$ \\
\hline Total & 100 & & & 100 & & \\
\hline \multicolumn{7}{|l|}{ Hand switch } \\
\hline No correction & $98.9(0.2)$ & - & & $16.4(2.9)$ & $511(40)$ & \\
\hline Instructed correction & $0.2(0.1)$ & - & - & $48.5(3.5)$ & $378(21)$ & $596(21)$ \\
\hline Wrong instructed correction & $0.4(0.1)$ & - & - & $3.4(1.0)$ & $521(47)^{c}$ & $849(100)^{c}$ \\
\hline Spontaneous correction & $0.3(0.1)$ & - & - & $11.9(2.0)$ & $420(33)^{d}$ & $390(49)^{d}$ \\
\hline Spontaneous correction + instructed correction & $0.1(0.0)$ & - & $-1-$ & $18.3(2.2)$ & $384(22)$ & $424(32) / 860(46)$ \\
\hline Spontaneous correction + wrong instructed correction & $0.0(0.0)$ & س. & $-1-$ & $1.5(0.3)$ & - & -1 \\
\hline Total & $100^{\mathrm{e}}$ & & & 100 & & \\
\hline
\end{tabular}

Note. RT1 and RT2 represent the latencies of the first and the second response, respectively. The latter was computed as the time elapsing between both responses. RT2 has two values when two corrections occurred. Spontaneous corrections are same-hand corrections in the hand-switch correction condition. Some mean latencies are based on a subsample of participants because these trial types did not occur in all participants. No mean latency was calculated when fewer than $75 \%$ of participants had valid data. The numbers in brackets are the standard errors of the mean. RT $=$ response time in milliseconds. ${ }^{\mathrm{a}} n=20 .{ }^{\mathrm{b}} n=19,{ }^{\mathrm{c}} n=18$. ${ }^{\mathrm{d}} n=21$. 'Deviation from 100 due to rounding.

\section{Results and Discussion}

The data were analyzed in the same way as in Experiment 1 . Again, trials with more than one correction response for one hand or with a response during the cue-stimulus interval $(<1 \%)$, and response time outliers $(1.3 \%)$ were removed. Moreover, two par-

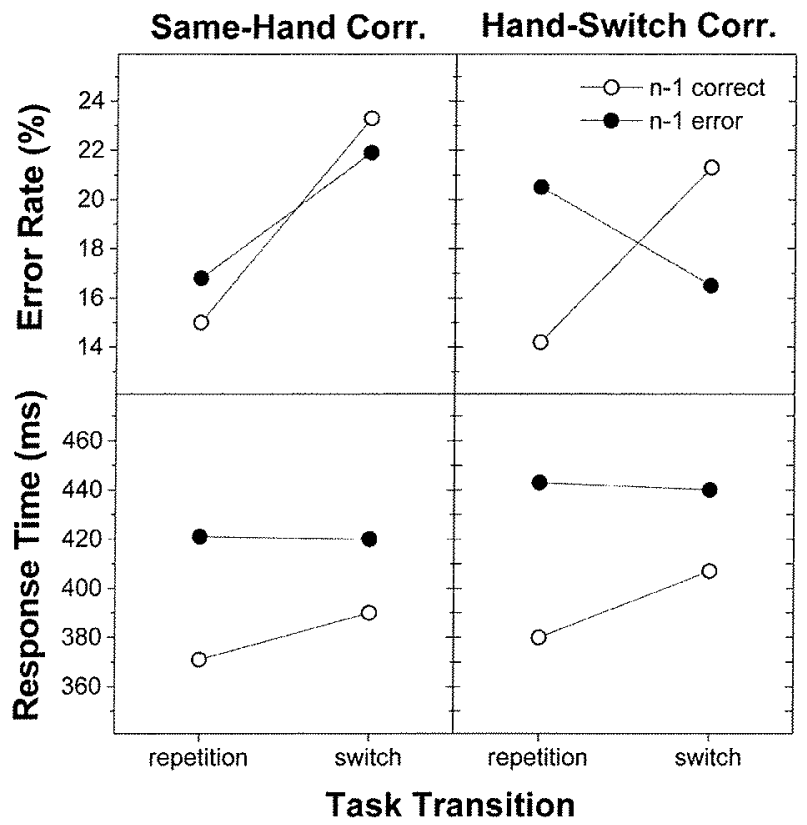

Figure 1. Error rates and response times for task switches and task repetitions following correct and erroneous responses in the same-hand correction condition and hand-switch correction condition in Experiment 1. Note that only trials following errors corrected by the instructed correction response were used. Corr. $=$ correction. ticipants were excluded because they repeatedly used the wrong hand for task responses or because the error rate was too low $(<5 \%)$ for the analyses. As shown in Table 2, the remaining participants reliably adhered to the instructions.

Again, only certain trial types were included in the main analyses. From the no-correction condition, I included only trials following uncorrected correct responses $(n-1$ correct) and trials following uncorrected errors ( $n-1$ error). From the hand-switch correction condition, I included only trials following uncorrected correct responses ( $n-1$ correct) and trials following errors that were only corrected by means of an effector switch ( $n-1$ error). Response times of correct responses and error rates of these trials were entered into a three-way ANOVA with repeated measures on the variables correction condition (no correction, hand-switch correction), task transition (repetition, switch), and $n-1$ accuracy ( $n-1$ correct, $n-1$ error). The results are shown in Figure 2. For the error rates, only the interaction between task transition and $n-$ 1 accuracy reached significance. Switch costs (repetition: 12.9\%; switch: $19.1 \%$ ) were obtained following correct responses, but switch benefits (repetition: $16.7 \%$; switch: $14.4 \%$ ) were obtained following errors, $F(1,19)=24.26, p<.001$. Similarly, the same interaction was marginally significant for the response times, indicating small switch costs (repetition: $389 \mathrm{~ms}$; switch: $405 \mathrm{~ms}$ ) following correct responses but switch benefits (repetition: 452 ms; switch: $428 \mathrm{~ms}$ ) following errors, $F(1,19)=3.57, p<.10$. In both analyses, this interaction was not further qualified by correction condition $(F \mathrm{~s}<1)$.

Taken together, the data revealed that errors corrected by handswitch corrections were followed by the same switch benefits as uncorrected errors. This suggests that hand-switch corrections imply no strengthening of the correct task as same-hand corrections do. These results further imply that the results of Experiment 1 are not due to a within-subject manipulation of correction type. Experiment 3 of Steinhauser and Hübner (2006) compared withinhand corrections with a no-correction condition in the same way 
Table 2

Relative Frequencies, Mean Response Times, and Correction Latencies of Trials With Different Correction Types for Errors and Correct First Responses Within the No-Correction Condition and Hand-Switch Correction Condition, Experiment 2

\begin{tabular}{|c|c|c|c|c|c|c|}
\hline \multirow[b]{2}{*}{ Correction } & \multicolumn{3}{|c|}{ Correct } & \multicolumn{3}{|c|}{ Error } \\
\hline & Frequency & RT1 & RT2 & Frequency & RT1 & RT2 \\
\hline \multicolumn{7}{|l|}{ None } \\
\hline No correction & $99.1(0.4)$ & $393(13)$ & & $76.3(4.4)$ & $392(22)$ & \\
\hline Spontaneous correction & $0.9(0.4)$ & $415(30)^{a}$ & $333(75)^{\mathrm{a}}$ & $23.7(4.4)$ & $402(24)$ & $519(75)$ \\
\hline Total & 100 & & & 100 & & \\
\hline \multicolumn{7}{|l|}{ Hand switch } \\
\hline No correction & $97.5(0.8)$ & $395(15)$ & & $13.1(2.2)$ & $600(63)^{b}$ & \\
\hline Instructed correction & $0.5(0.2)$ & - & - & $63.7(4.4)$ & $385(20)$ & $649(20)$ \\
\hline Wrong instructed correction & $0.7(0.4)$ & - & - & $3.8(1.5)$ & - & - \\
\hline Spontaneous correction & $0.7(0.4)$ & - & - & $4.6(1.0)$ & $442(39)^{\circ}$ & $387(68)^{4}$ \\
\hline Spontaneous correction + instructed correction & $0.1(0.0)$ & - & $-1-$ & $13.1(2.7)$ & $439(31)^{d}$ & $365(45)^{d / 855(45)}$ \\
\hline Spontaneous correction + wrong instructed correction & $0.1(0.1)$ & - & $-1-$ & $1.7(0.8)$ & - & $-1-$ \\
\hline Total & $100^{\mathrm{c}}$ & & & $100^{c}$ & & \\
\hline
\end{tabular}

Note. RT1 and RT2 represent the latencies of the first and the second response, respectively. The latter was computed as the time elapsing between both responses. RT2 has two values when two corrections occurred. Spontaneous corrections are same-hand corrections. Some mean latencies are based on a subsample of participants because these trial types did not occur in all participants. No mean latency was calculated when fewer than $75 \%$ of participants had valid data. The numbers in brackets are the standard errors of the mean. $\mathrm{RT}=$ response time in milliseconds.

${ }^{\mathrm{a}} n=15 .{ }^{\mathrm{b}} n=18 .{ }^{\mathrm{c}} n=17 .{ }^{\mathrm{d}} n=19 .{ }^{\mathrm{c}}$ Deviation from 100 due to rounding.

that the present Experiment 2 compared hand-switch corrections with a no-correction condition. Because the result pattern across these two experiments resembles that of Experiment 1, the present results seem not to depend on whether correction type is manipulated within-subjects or between-subjects.

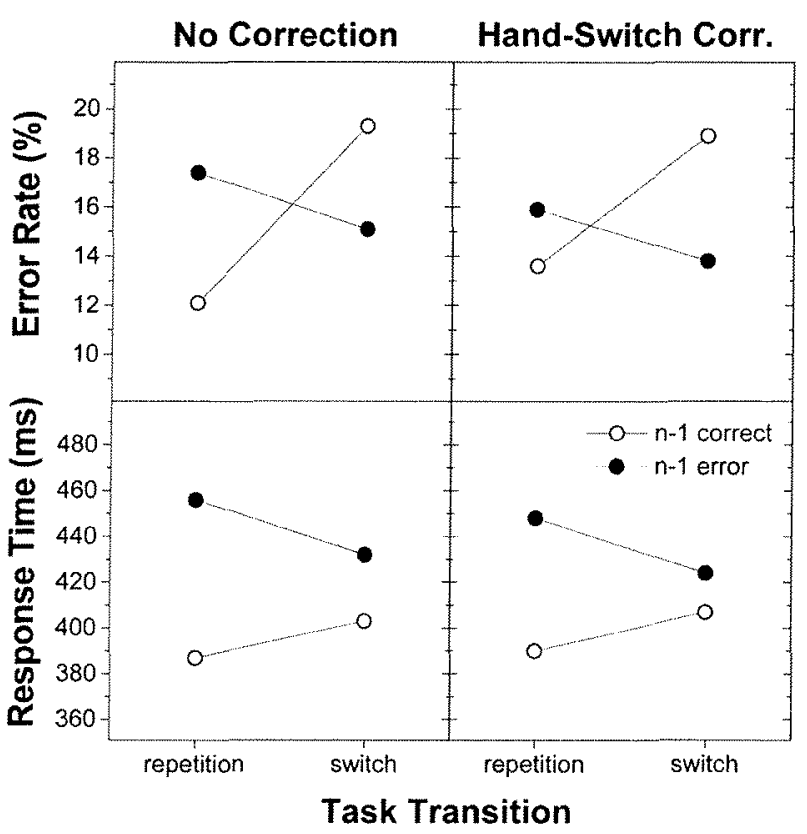

Figure 2. Error rates and response times for task switches and task repetitions following correct and erroneous responses in the no-correction condition and same-hand correction condition in Experiment 2. Note that only trials following uncorrected errors were used in the no-correction condition and only trials following corrected errors were used in the same-hand correction condition. Corr. = correction.

\section{Experiment 3}

The results of the first two experiments suggest that activating response categories such as left or right during error correction is not sufficient to trigger strengthening of the correct task and, thus, to turn switch benefits back into switch costs. However, the question emerges as to whether hand-switch correction really involved the same response categories as correct responses. Although the task instruction emphasized the categories left and right and these spatial codes are known to be preferentially used in this type of task (Druey \& Hübner, 2008), it is possible that at least some participants represented their responses in terms of the fingers (e.g., index finger, middle finger). In this case, handcorrections might have failed to trigger strengthening simply because response categories had to be recoded during correction (e.g., if middle finger is the correct response, the correction requires index finger). To rule out such an explanation, Experiment 2 was replicated with a different correction procedure.

In Experiment 3, participants were instructed to correct their errors by vocalizing the correct response category ("left," "right"). This should force participants to use spatial codes as response categories, and, indeed, it has been shown that manual and vocal left/right responses produce cross-modal category repetition effects (Schuch \& Koch, 2004). However, if only task-relevant responses trigger strengthening, then even vocal corrections should be followed by the same switch benefits as uncorrected errors.

\section{Method}

Twenty participants ( 16 women, 4 men) between 20 and 36 years of age (mean age 23.1 years) with normal or corrected-tonormal vision participated in the study. The design and procedure of the experiment were the same as in Experiment 2. However, the hand-switch correction condition was replaced by a vocal correction condition, in which participants were instructed to correct their errors by overtly vocalizing the correct response category 
"left" or "right." Vocal correction responses were recorded and classified offline. Response onsets were determined online via a threshold procedure implemented in Presentation 12.1 (Neurobehavioral Systems, Inc.).

\section{Results and Discussion}

Again, trials with more than one correction response or with a response during the cue-stimulus interval $(<1 \%)$, and response time outliers $(1.1 \%)$ were removed. Table 3 shows the frequencies of correction responses following correct and error responses in each correction condition. The rate of vocally corrected errors (69\%) was comparable to that of hand-switch corrections in the previous experiments.

The data were analyzed in the same way as in Experiment 2. The results are shown in Figure 3. For the error rates, only the interaction between task transition and $n-1$ accuracy reached significance. Switch costs (repetition: 13.8\%; switch: 19.8\%) were obtained following correct responses, but small switch benefits (repetition: $17.1 \%$; switch: $16.4 \%$ ) were obtained following errors, $F(1,19)=7.34, p<.05$. However, Figure 3 reveals that, although the three-way interaction was not significant, $F(1,19)=2.30, p<$ .15 , switch benefits were obtained only following vocally corrected errors. In contrast, uncorrected errors were now followed by small switch costs. For the response times, only a marginally significant interaction between task transition and $n-1$ accuracy was obtained, $F(1,19)=4.01, p<.10$, indicating a similar trend as in the error rates.

Taken together, these results show that correcting errors vocally is not sufficient to trigger strengthening of the correct task and, thus, to produce the same switch costs on the subsequent trial as correct responses. Moreover, at least statistically, switch effects following vocally corrected errors were similar to those following uncorrected errors. However, it is surprising that only vocally corrected errors but not uncorrected errors caused numerical

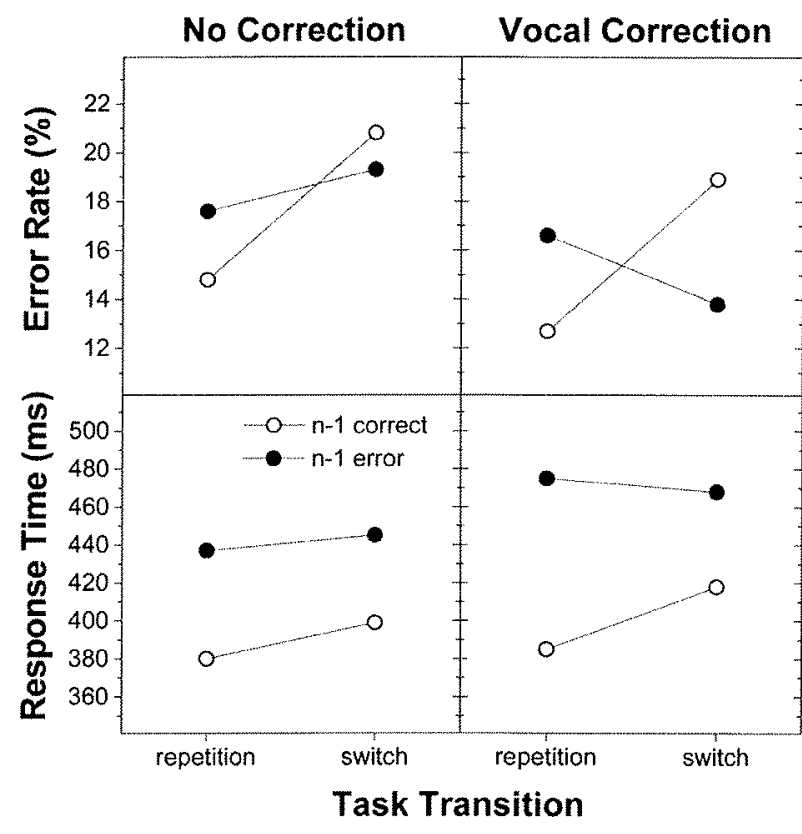

Figure 3. Error rates and response times for task switches and task repetitions following correct and erroneous responses in the no-correction condition and the vocal correction condition in Experiment 3. Note that only trials following uncorrected errors were used in the no-correction condition and only trials following corrected errors were used in the vocal correction condition.

switch benefits on the subsequent trial. This clearly contradicts the results of earlier studies examining the effect of uncorrected errors on subsequent switch effects (Steinhauser \& Hübner, 2006, 2008). Possibly, participants had to actively suppress vocal corrections in

Table 3

Relative Frequencies, Mean Response Times, and Correction Latencies of Trials With Different Correction Types for Errors and Correct First Responses Within the No-Correction Condition and Vocal-Correction Condition, Experiment 3

\begin{tabular}{|c|c|c|c|c|c|c|}
\hline \multirow[b]{2}{*}{ Correction } & \multicolumn{3}{|c|}{ Correct } & \multicolumn{3}{|c|}{ Error } \\
\hline & Frequency & RT1 & $\mathrm{RT} 2$ & Frequency & RT1 & RT2 \\
\hline \multicolumn{7}{|l|}{ None } \\
\hline No correction & $99.5(0.2)$ & $388(18)$ & & $80.8(3.2)$ & $402(42)$ & \\
\hline Spontaneous correction & $0.5(0.2)$ & - & - & $19.2(3.2)$ & $388(18)$ & 397 (74) \\
\hline Total & 100 & & & 100 & & \\
\hline \multicolumn{7}{|l|}{ Vocal } \\
\hline No correction & $98.5(0.4)$ & $402(19)$ & & $21.3(3.4)$ & $540(59)$ & \\
\hline Instructed correction & $0.2(0.1)$ & - & - & $64.5(3.3)$ & $378(17)$ & $748(33)$ \\
\hline Wrong instructed correction & $1.0(0.4)$ & $\ldots$ & - & $3.3(1.8)$ & - & - \\
\hline Spontaneous correction & $0.3(0.1)$ & - & - & $6.7(1.9)$ & $412(24)^{a}$ & $427(140)^{a}$ \\
\hline Spontaneous correction + instructed correction & $0.0(0.0)$ & - & $-1-$ & $4.0(1.0)$ & $379(15)^{b}$ & $150(17)^{b / 852(37)^{b}}$ \\
\hline Spontaneous correction + wrong instructed correction & $0.0(0.0)$ & - & $-1-$ & $0.4(0.2)$ & - & -1 \\
\hline Total & 100 & & & $100^{\mathrm{c}}$ & & \\
\hline
\end{tabular}

Note. RT1 and RT2 represent the latencies of the first and the second response, respectively. The latter was computed as the time elapsing between both responses. RT2 has two values when two corrections occurred. Spontaneous corrections are same-hand corrections. Some mean latencies are based on a subsample of participants because these trial types did not occur in all participants. No mean latency was calculated when fewer than $75 \%$ of participants had valid data. The numbers in brackets are the standard errors of the mean. $\mathrm{RT}=$ response time in milliseconds.

${ }^{\mathrm{a}} n=17 .{ }^{\mathrm{b}} n=16 .{ }^{\mathrm{c}}$ Deviation from 100 due to rounding. 
the no-correction condition, which might have abolished any switch effects on the subsequent trial. Such active suppression was not necessary in Experiment 2 because participants could remove their correction hand from the keyboard during the no-correction condition.

\section{General Discussion}

The goal of the present study was to investigate the conditions necessary for triggering task strengthening and producing switch costs in task switching. To this end, this study examined which type of correction response is necessary to reverse strengthening of the wrong task on error trials. Same-hand corrections required that errors be corrected with the same effectors that were used for the main tasks. In contrast, hand-switch corrections required that errors be corrected with the hand not used for the main tasks and vocal corrections required that the correct response category be vocalized. If only task-relevant responses trigger task strengthening, then only same-hand corrections should be followed by switch costs (as found by Steinhauser \& Hübner, 2006), whereas handswitch corrections and vocal corrections should be followed by switch benefits on the subsequent trial. However, if the selection of abstract response categories (such as left or right) is sufficient to trigger strengthening, then each type of correction response should trigger strengthening of the correct task and produce switch costs on the subsequent trial. The results indicate that only same-hand corrections led to subsequent switch costs. Experiment 1 showed that same-hand corrections but not hand-switch corrections led to switch costs following errors. Experiment 2 showed that handswitch corrections produced similar switch benefits after errors as uncorrected errors. Finally, Experiment 3 showed that vocal corrections also produced switch benefits following errors.

Taken together, the present results suggest that only the execution of a task-relevant response but not the selection of abstract response categories triggers strengthening. The question emerges as to why such a strengthening mechanism makes sense. One possibility is that task strengthening serves the automatization of tasks (cf. the idea of micropractice; Meiran, 1996). Normally, this mechanism leads to a continuous improvement of performance in the course of repeated practice. However, when two tasks make use of the same responses and stimuli, then strengthening one task implies that the other task is weakened. In this case, task strengthening leads to costs in case of a task switch.

The idea that task strengthening is related to automatization could also explain why strengthening is triggered by task-televant responses only. Efficient automatization of a specific task requires that strengthening occur when this task is maximally active. This can be achieved when strengthening is triggered by the occurrence of a task-relevant response because the response is a valid cue for the relevant task being strongly active. If other responses were also able to trigger strengthening-for example, because they share the same response categories with the task-relevant responses-this would increase the risk of strengthening wrong associations or of strengthening at a time when the relevant task is not strongly active. Thus, the fact that only task-relevant responses trigger strengthening could be a fundamental constraint that enables optimal automatization of tasks. A question that is still open is why task strengthening works in an unsupervised way-that is, why error detection does not prevent the wrong task from being strengthened at all. This could reflect the fact that error detection in choice tasks often occurs several hundred milliseconds after the response (Steinhauser et al., 2008) and thus cannot prevent that strengthening of the wrong task occurs. This situation implies that learning and task optimization are not necessarily guided by performance monitoring, as suggested by recent theories (e.g., Brown, Reynolds, \& Braver, 2007).

A possible objection to the present conclusions could result from the observation that same-hand corrections and hand-switch corrections also differ with respect to correction latency-that is, the time elapsed between the first response and the correction response. The difference in correction latency has two possible sources that offer alternative explanations for the present results: First, hand-switch corrections could require an additional timeconsuming processing stage in which the correct response (derived in the course of error detection; Steinhauser et al., 2008) is translated into a correction response for the other hand. This is not necessary in same-hand corrections because the correction response is identical with the correct response. Second, some of the same-hand corrections are probably automatic and fast error corrections, leading to a reduction of mean correction latency.

The idea of an additional processing stage in hand-switch corrections could account for the present results if one assumes that, during the delay caused by this stage, activation of the correct task can decay. Given that less activation should imply less strengthening (a typical assumption in Hebbian learning), task strengthening would still be triggered but would be less effective. Affirming against such an explanation is the finding by Philipp et al. (2007) that switch costs on the subsequent trial are also obtained when the preceding response is considerably delayed by means of a delayed go signal. Alternatively, the results could reflect the fact that some of the same-hand corrections are automatic corrections and that only these automatic corrections imply strengthening of the correct task. However, Steinhauser and Hübner (2006) analyzed switch effects following spontaneous corrections in a condition where corrections were discouraged. The switch costs following these corrections were rather small and not significant, which clearly speaks against the idea that only fast and automatic corrections imply task strengthening.

Another objection could be that, in contrast to same-hand corrections, hand-switch corrections and vocal corrections require a switch of response set, which itself can be viewed as a task switch (Koch, Gade, \& Philipp, 2004; Philipp \& Koch, 2005). In this case, trials following these error corrections would be always either partial task-switch trials (i.e., only the response set is switched) or full task-switch trials (i.e., response set and task rules are switched). However, this could not explain why a switch benefit, that is, a benefit of full task switches relative to partial task switches, is obtained following these trials. At least with a long cue-stimulus interval, partial task switches and full task switches have been shown to produce similar switch costs (for a recent overview, see Vandierendonck, Christiaens, \& Liefooghe, 2008). Accordingly, the present results suggest that error corrections are not represented as separate task sets and thus do not require a task switch.

In summary, the present study provides evidence that only the execution of task-relevant responses can trigger task strengthening. This finding not only supports response-based strengthening as a mechanism underlying switch effects in task switching, but it also 
provides important insights into the functional role of task strengthening. Specifically, this finding suggests that task strengthening is related to the automatization of effector-specific cognitive skills.

The present study has also some implications regarding the relation between task-switch effects and intentional control processes. Although the task switching paradigm has initially been used as a paradigm to investigate intentional control (Rogers \& Monsell, 1995), recent accounts have assumed that not only a portion but rather all switch costs reflect priming-that is, an aftereffect of recent processing (e.g., Altmann \& Gray, 2008; Logan \& Bundesen, 2003). But even in this case, one could still ask whether the costs are at least an aftereffect of intentional processing and, thus, can be used as an indirect indicator of control processes. The present results (as well as the results of Steinhauser \& Hübner, 2006) are not consistent with such a view. By demonstrating that switch effects reflect erroneously applied tasks instead of intended tasks, the results show that switch effects are an aftereffect of unintended and merely incidental processing.

\section{References}

Allport, A., Styles, E. A., \& Hsieh, S. (1994). Shifting intentional set: Exploring the dynamic control of tasks. In C. Umiltà \& M. Moscovitch (Eds.), Attention and performance XV: Conscious and nonconscious information processing (pp. 421-452). Cambridge, MA: MIT Press.

Altmann, E. M., \& Gray, W. D. (2008). An integrated model of cognitive control in task switching. Psychological Review, 115, 602-639.

Brown, J. W., Reynolds, J. R., \& Braver, T. S. (2007). A computational model of fractionated conflict-control mechanisms in task-switching. Cognitive Psychology, 55, 37-85.

Druey, M., \& Hübner, R. (2008). Effects of stimulus features and instruction on response coding, selection, and inhibition: Evidence from repetition effects under task switching. Quarterly Journal of Experimental Psychology, 61, 1573-1600.

Fiehler, K., Ullsperger, M., \& Von Cramon, D. Y. (2005). Electrophysiological correlates of error correction. Psychophysiology, 42, 72-82.

Hübner, R., \& Druey, M. D. (2006). Response execution, selection, or activation: What is sufficient for response-related repetition effects under task shifting? Psychological Research, 70, 245-261.

Koch, I., Gade, M., \& Philipp, A. M. (2004). Inhibition of response mode in task switching. Experimental Psychology, 51, 52-58.

Logan, G. D., \& Bundesen, C. (2003). Clever homunculus: Is there an endogenous act of control in the explicit task-cuing procedure? Journal of Experimental Psychology: Human Perception and Performance, 29, 575-599.

Meiran, N. (1996). Reconfiguration of processing mode prior to task performance. Journal of Experimental Psychology: Learning, Memory, and Cognition, 22, 1423-1442.

Meiran, N. (2000). Modeling cognitive control in task-switching. Psychological Research, 63, 234-249.

Meiran, N., \& Daichman, A. (2005). Advance task preparation reduces task error rate in the cuing task-switching paradigm. Memory \& Cognition, $33,1272-1288$.

Philipp, A. M., Jolicoeur, P., Falkenstein, M., \& Koch, I. (2007). Response selection and response execution in task switching: Evidence from a go-signal paradigm. Journal of Experimental Psychology: Learning Memory, and Cognition, 33, 1062-1075.

Philipp, A. M., \& Koch, I. (2005). Switching of response modalities. The Quarterly Joumal of Experimental Psychology: Human Experimental Psychology, 58(A), 1325-1338.

Rogers, R. D., \& Monsell, S. (1995). Costs of a predictable switch between simple cognitive tasks. Joumal of Experimental Psychology: General, 124, 207-231

Schuch, S., \& Koch, I. (2003). The role of response selection for inhibition of task sets in task shifting. Journal of Experimental Psychology: Human Perception and Performance, 29, 92-105.

Schuch, S., \& Koch, I. (2004). The costs of changing the representation of action: Response repetition and response-response compatibility in dual tasks. Joumal of Experimental Psychology: Human Perception and Performance, 30, 566-582.

Steinhauser, M., \& Hübner, R. (2006). Response-based strengthening in task shifting: Evidence from shift effects produced by errors. Joumal of Experimental Psychology: Human Perception and Performance, 32, 517-534.

Steinhauser, M., \& Hübner, R. (2008). How task errors affect subsequent behavior: Evidence from distributional analyses of task-switching effects. Memory \& Cognition, 36, 979-990.

Steinhauser, M., Maier, M., \& Hübner, R. (2008). Modeling behavioral measures of error detection in choice tasks: Response monitoring versus conflict monitoring. Journal of Experimental Psychology: Human Per ception and Performance, 34, 158-176.

Vandierendonck, A., Christiaens, E., \& Liefooghe, B. (2008), On the representation of task information in task switching: Evidence from task and dimension switching. Memory \& Cognition, 36, 1248-1261.

Winer, B. J., Brown, D. R., \& Michels, K. M. (1991). Statistical principles in experimental design. New York, NY: McGraw-Hill. 